\title{
ANÁLISE DOS EFEITOS GEOMÉTRICOS DE ENTRADA E SAÍDA EM CICLONES POR SIMULAÇÃO NUMÉRICA E EXPERIMENTOS FÍSICOS
}

\author{
E. BALESTRIN, R. D. LUCIANO, V. R. WIGGERS, R.K. DECKER, H. F. MEIER \\ Universidade Regional de Blumenau, Departamento de Engenharia Química
}

\begin{abstract}
RESUMO - A partir de experimentos e simulações numéricas, realizou-se um estudo dos efeitos geométricos de entrada e saída em ciclones com validação de modelo. As simulações foram realizadas com modelo de turbulência RSM (Reynolds Stress Model) em código comercial de fluidodinâmica computacional (CFD). Como domínio físico, utilizou-se duas configurações geométricas, uma com o ciclone e outra contendo os dispositivos de entrada e saída e seus prolongamentos. Os experimentos foram realizados em uma bancada experimental de dutos e ciclones com o auxílio de manômetro diferencial para medida da perda de carga, e com velocimetria por imagem de partícula estereoscópica para medida dos perfis de velocidade. Observou-se uma boa representação numérica da perda de carga em diferentes condições operacionais, e um comportamento diferenciado no campo de velocidade no ciclone em relação aos ciclones convencionais, atribuído aos efeitos de entrada e saída, com a existência de um vórtice descendente adicional como resultado da reversão do escoamento na saída do ciclone.
\end{abstract}

\section{INTRODUÇÃO}

Ciclones são equipamentos que comportam uma variedade de aplicações, tais como separação gás-sólido e gás líquido, reatores, dispositivos de troca térmica, entre outras. Quando aplicados para separação gás-sólido, os mesmos fazem uso da força centrífuga para separar as partículas da fase gasosa, a qual serve como transporte do material particulado. É o equipamento mais utilizado nas indústrias quando se trata de coleta de partículas na faixa de 5 a $200 \mu \mathrm{m}$. Esta preferência ocorre devido a sua boa relação custo-benefício, onde consegue-se incluir em um mesmo dispositivo, vantagens como simplicidade de construção, facilidade de manutenção e larga faixa de operação de temperatura e pressão. Além disto, o mesmo ainda é capaz de alcançar altas eficiências de coleta para uma determinada faixa granulométrica de particulados. Em contrapartida, a fluidodinâmica do escoamento turbulento em ciclones é complexa, com fenômenos que incluem regiões de circulação, zonas de reversão do escoamento, contato gás-sólido, entre outros.

De acordo com Hoffmann e Stein (2008) a performance de ciclones é geralmente descrita em relação aos seus parâmetros de desempenho, como a perda de carga e a eficiência de coleta. Para se entender e melhorar os mecanismos de perda de pressão e de separação das partículas, é importante compreender o escoamento da fase gasosa no interior do ciclone. E para isso é necessário resolver as equações gerais que governam o escoamento (equações de conservação da 
massa e do momentum), que independem das dimensões dos ciclones através de técnicas numéricas, utilizando a fluidodinâmica computacional (EL-BATSH, 2012). A validação dos resultados numéricos é realizada por meio de dados experimentais adquiridos com sondas de pressão, medidores de temperatura, velocimetria por imagem de partícula (PIV), anemometria de fio e filme quente, entre outros.

Os parâmetros de desempenho dos ciclones estão relacionados com suas condições operacionais (campos de velocidade e pressão, distribuição granulométrica das partículas, propriedades físicas das fases gasosa e sólida), e com sua configuração geométrica. Diversos estudos em CFD já foram realizados na tentativa de entender e melhorar o escoamento giratório observado no interior dos ciclone, e por conseguinte seus parâmetros de desempenho. Dentre esses pode-se citar os realizados: por Noriler et al. (2004), com a proposição de uma configuração geométrica no duto de saída do gás em formato de espiral, com a finalidade de reduzir a perda de carga; por Bernardo et al. (2005), com estudo sobre a influência da modificação do ângulo da seção de entrada do ciclone em relação ao seu corpo cilíndrico; por Dias (2009), que buscou variar quatro configurações distintas na região de saída de sólidos e a influência do modelo de turbulência sobre a predição do escoamento ciclônico; por El-Batsh (2012), com análise dos efeitos do diâmetro e do comprimento do duto de saída de gás sobre a perda de carga e a eficiência de coleta; por Cernecky e Plandorova (2013), sobre o efeito da introdução do duto de saída do gás para o interior do corpo cilíndrico do ciclone sobre a performance de ciclones; e por Sgrott Jr. et al. (2013) que realizou um estudo de otimização geométrica de ciclones por combinação de técnicas de CFD e de otimização.

Porém, nenhum estudo sobre os efeitos geométricos dos dispositivos antes e após o ciclone (entrada e saída) sobre o escoamento giratório no interior dos ciclones foram encontrados na literatura. Desta forma, este estudo tem como objetivo analisar os efeitos geométricos de entrada e saída em ciclones por experimentações físicas e simulações numéricas com validação de modelos.

\section{MATERIAIS E MÉTODOS}

\subsection{Experimentos Físicos}

Os experimentos físicos foram realizados na Unidade Experimental de Dutos e Ciclones (UE-DC), ilustrada na Figura 1. Realizou-se experimentos de perda de carga para o escoamento monofásico variando-se a velocidade de entrada em uma faixa de 8 a $16 \mathrm{~m} / \mathrm{s}$, e experimentos com o sistema de velocimetria por imagem de partícula estereoscópica (Stereo PIV) para obter os campos de velocidade no interior do ciclone. Para as medidas com Stereo PIV utilizou-se uma partícula com diâmetro de Sauter de $3,89 \mu \mathrm{m}$ e massa especifica de $1400 \mathrm{~kg} / \mathrm{m}^{3}$ como traçadora do escoamento da fase gasosa, e velocidade na entrada do ciclone em $12 \mathrm{~m} / \mathrm{s}$. Os experimentos de perda de carga foram repetidos dez vezes, e os experimentos de obtenção dos campos de velocidade foram realizados em triplicata, para com isso avaliar e quantificar as incertezas experimentais.

A configuração utilizada para obtenção dos dados experimentais relacionados ao escoamento monofásico, consiste em um conjunto de tubulações de admissão da fase gasosa, passando pelo ciclone de acrílico (05) (dimensões em mm) até sair no filtro manga (09). Para os experimentos de 
perda de carga, tem-se um medidor diferencial de pressão (04) acoplado ao ciclone, que obtêm as medidas de perda de carga. Já para a obtenção dos campos de velocidade no interior do ciclone, utiliza-se o sistema Stereo PIV (08), o laser (03) e o alimentador das partículas traçadoras (01).

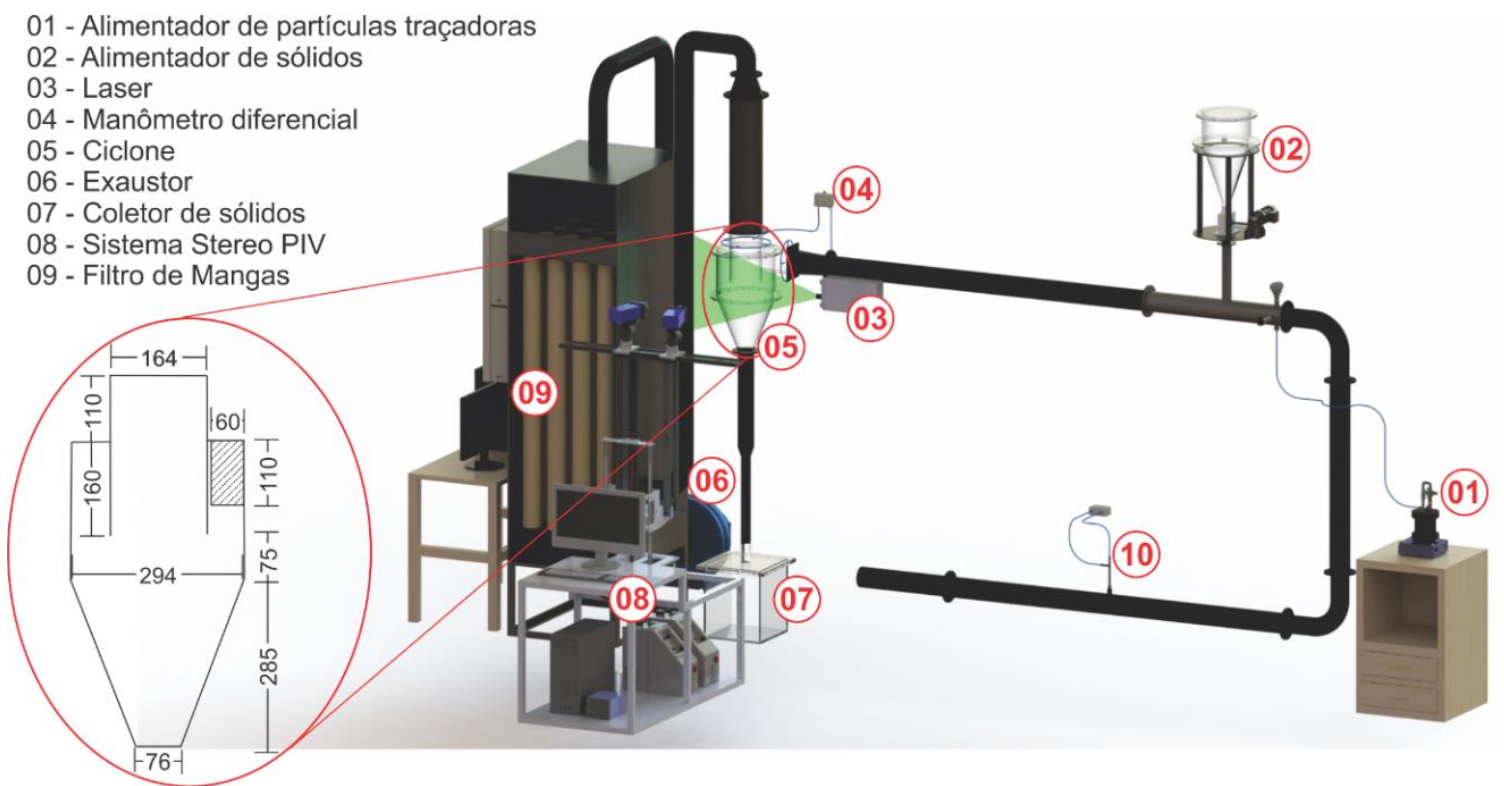

Figura 1: Unidade Experimental de Dutos e Ciclones com as dimensões do ciclone em (mm).

\subsection{Simulações Numéricas}

As simulações numéricas foram realizadas em código comercial FLUENT 14.0 da ANSYS. Este é um código numérico de CFD que possibilita a simulação de configurações geométricas variadas. Portanto, as simulações foram realizadas a partir de duas geometrias distintas, uma delas com toda a configuração geométrica da UE-DC (Figura 2 (a)), e outra, apenas com a geometria do ciclone acoplado a caixa coletora (Figura 2 (b)).
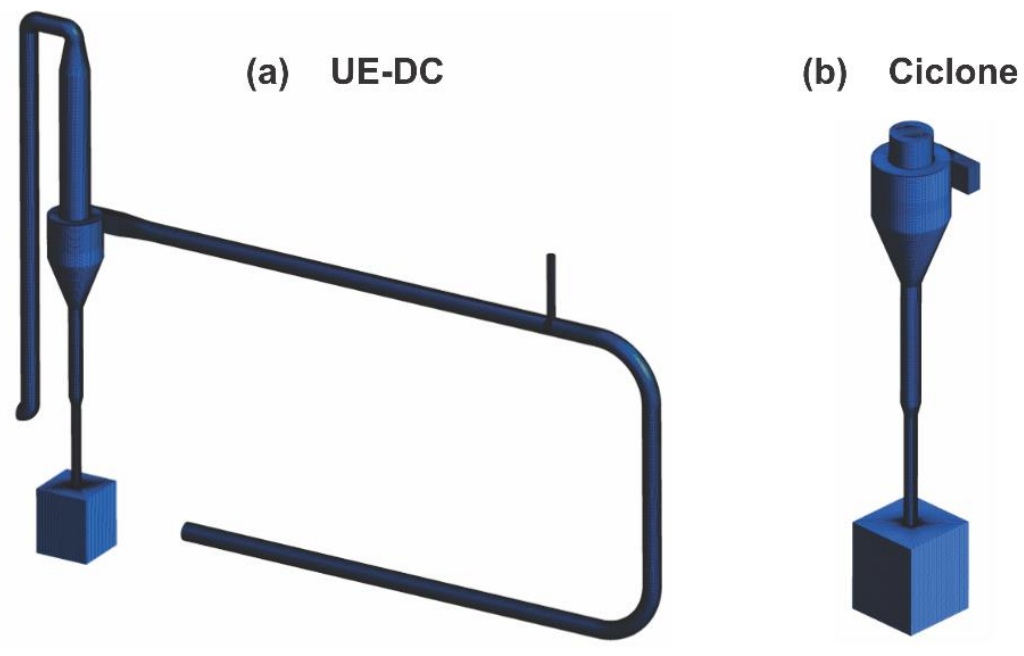

Figura 2: Geometrias utilizadas nas simulações numéricas. 
As simulações foram realizadas com modelo de turbulência "RSM-SSG", acoplamento pressão velocidade "SIMPLEC" e discretização espacial: da pressão por algoritmo "PRESTO", do movimento com esquema "Second Order Upwind", e da energia cinética turbulenta, taxa de energia cinética turbulenta com "First Order Upwind", além da formulação explicita no tempo.

A partir das simulações obteve-se dados de perda de carga e perfis de velocidade axial e tangencial em região localizada no corpo cilíndrico do ciclone.

\section{MODELAGEM MATEMÁTICA}

A modelagem matemática proposta, segue os princípios ordenadores da natureza, como o princípio da conservação da massa e da quantidade de movimento. Esta é representada pelas equações de Navier-Stokes, abordadas do ponto de vista das médias temporais de Reynolds (RANS) e sob enfoque Euleriano.

Conservação da massa,

$$
\frac{\partial}{\partial t}\left(\rho_{g}\right)+\nabla \cdot\left(\rho_{g} v_{g}\right)=0
$$

Conservação da quantidade de movimento,

$$
\frac{\partial}{\partial t}\left(\rho_{g} \boldsymbol{v}_{g}\right)+\nabla \cdot\left(\rho_{g} \boldsymbol{v}_{g} \boldsymbol{v}_{g}\right)=-\nabla \cdot\left(\boldsymbol{T}_{g}^{e f f}\right)+\rho_{g} \boldsymbol{g}-\nabla p
$$

Onde o subscrito "g" representa a fase gasosa e o tensor efetivo de Reynolds pode ser definido como a soma do tensor turbulento de Reynolds $\left(\mathbf{T}_{g}^{t}\right)$ e o tensor molecular $\left(\mathbf{T}_{\mathrm{g}}\right)$, ou seja:

$$
\boldsymbol{T}_{g}^{e f f}=\boldsymbol{T}_{g}^{t}+\boldsymbol{T}_{g}
$$

Para o fechamento da turbulência utilizou-se o modelo (RSM). A modelagem RSM faz o fechamento das equações RANS (Reynolds-Averaged Navier-Stokes) resolvendo as equações de transporte de Reynolds, juntamente com uma equação para a taxa de dissipação $\left(\varepsilon_{\mathrm{g}}\right)$. Isto significa que são requeridas sete equações de transporte adicionais para resolver o escoamento da fase gasosa. A equação de conservação para o tensor de Reynolds é expressa por:

$$
\frac{\partial}{\partial \mathrm{t}}\left(\rho_{\mathrm{g}} \boldsymbol{T}_{g}^{t}\right)+\nabla \cdot \mathbf{v}_{\mathrm{g}}\left(\rho_{\mathrm{g}} \boldsymbol{T}_{g}^{t}\right)=\mathbf{D}_{\mathbf{T}}+\mathbf{D}_{\mathbf{M}}+\boldsymbol{Y}+\boldsymbol{\Pi}-\frac{2}{3} \boldsymbol{\delta} \rho_{\mathrm{g}} \varepsilon_{\mathrm{g}}
$$

Dos vários termos da equação do modelo RSM, os termos referente a difusão molecular $\left(\mathbf{D}_{\mathbf{M}}\right)$ e a taxa de produção ( $\mathbf{Y}$ ), não requerem nenhuma modelagem. No entanto, os termos referentes a difusão turbulenta $\left(\mathbf{D}_{\mathbf{T}}\right)$ e a deformação devido a pressão $(\boldsymbol{\Pi})$ precisam ser modelados para 0 fechamento das equações. $O$ termo correspondente a taxa de deformação devido a pressão (П) pode ser definido de diferentes formas. Neste trabalho abordou-se a modelagem quadrática proposta por Speziale et al. (1991), a qual é referenciada como RSM-SSG. 


\section{RESULTADOS E DISCUSSÕES}

\subsection{Teste de malha}

O teste de malha é importante para a definição da concentração da malha numérica mais apropriada ao estudo de caso, afim de minimizar os erros de discretização. Realizou-se um teste de malha com 3 malhas distintas, conforme Figura 3. Deste teste, observou-se que os perfis de velocidade tangencial não apresentam diferenças significativas entre as malhas simuladas, no entanto, quando analisado o perfil de velocidade axial, observou-se que o refinamento da malha traz consequências aos picos da velocidade axial. Sendo assim, optou-se por utilizar a malha com maior refinamento. De modo que a malha para a geometria apenas do ciclone é a mesma da geometria da UE-DC, porém sem os dutos de entrada e saída.
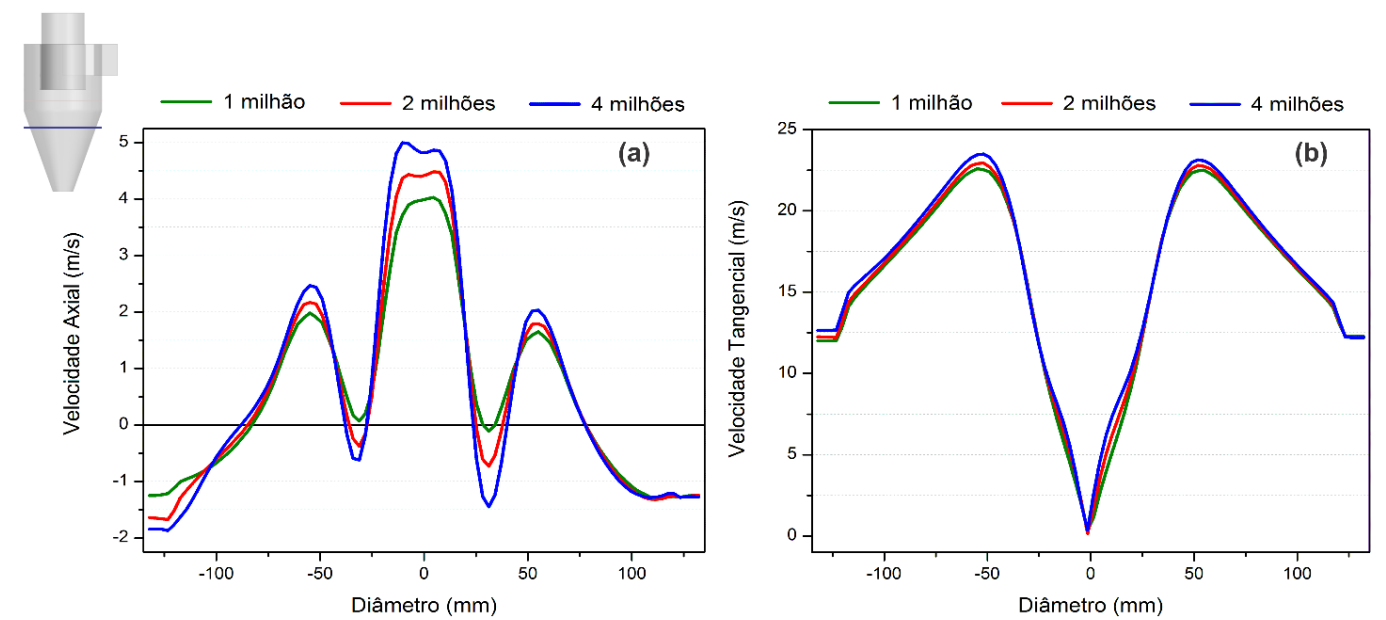

Figura 3: Teste de malha para a velocidade na entrada do ciclone em $12 \mathrm{~m} / \mathrm{s}$ : (a) perfis de velocidade axial; (b) perfis de velocidade tangencial.

\subsection{Estudos de validação da predição da perda de carga}

A validação do modelo de turbulência RSM-SSG foi realizada através da comparação dos resultados numéricos e experimentais. De modo que a validação do modelo turbulência foi realizada com base em dados de perda de carga e de perfis de velocidade axial e tangencial.

A Tabela 1 apresenta a perda de carga obtida através da média dos dez experimentos realizados, a perda de carga simulada para a geometria da UE-DC, e os erros absoluto e relativo da simulação numérica em relação aos dados experimentais. Para melhor ilustrar os resultados de perda de carga experimentais e simulados, gerou-se um gráfico, conforme Figura 4 (a), sendo que a barra do erro experimental apresentado, é a soma entre o desvio padrão dos dez experimentos e o erro de precisão do manômetro diferencial; e a barra do erro numérico representa a incerteza numérica calculada através do método GCI (Grid Convergence Index). A partir dos resultados, concluiu-se que o modelo de turbulência RSM-SSG representou bem os dados experimentais, uma vez que o erro relativo não excedeu os $8,25 \%$ para nenhuma das velocidades estudadas, e as barras da incerteza experimental e numérica se sobrepuseram. 


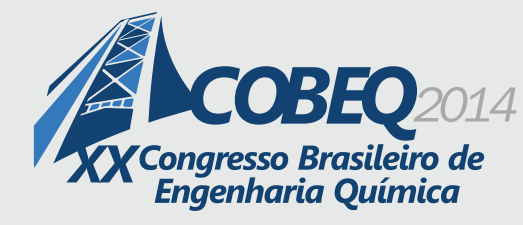

19 a 22 de outubro de 2014
Florianópolis/SC

Tabela 1: Resultados de perda de carga experimental e simulado para a geometria da UE-DC.

\begin{tabular}{c|cc|c|c}
\multirow{2}{*}{$\mathrm{v}(\mathrm{m} / \mathrm{s})$} & \multicolumn{2}{|c|}{ Perda de Carga $(\mathrm{Pa})$} & Erro Absoluto (Pa) & Erro Relativo (\%) \\
\cline { 2 - 5 } & Numérico (UE-DC) & Experimental & Numérico (UE-DC) & Numérico (UE-DC) \\
\hline 8 & 141,52 & 145,12 & 3,60 & 2,48 \\
\hline 10 & 223,23 & 233,60 & 10,37 & 4,44 \\
\hline 12 & 323,23 & 343,36 & 20,13 & 5,86 \\
\hline 14 & 440,21 & 477,22 & 37,01 & 7,76 \\
\hline 16 & 580,43 & 632,64 & 52,21 & 8,25 \\
\hline
\end{tabular}

Os efeitos geométricos de entrada e saída sobre a perda de carga em ciclones foi analisado através de simulações numéricas utilizando duas geometrias distintas (Figura 4 (b)). Destas, observou-se que a perda de carga simulada com a configuração geométrica da UE-DC, obteve uma perda de carga inferior a simulada com a geometria apenas do ciclone. Tal diminuição da perda de carga pode ser justificada pelo fato de o perfil de velocidade axial na entrada do ciclone, quando simulado a geometria da UE-DC, ser um perfil parabólico, e quando simulado apenas o ciclone, ser um perfil empistonado. Deste modo, no primeiro caso ocorre uma redução da intensidade do escoamento giratório, a qual é responsável pela maior parcela da perda de carga nos ciclones.
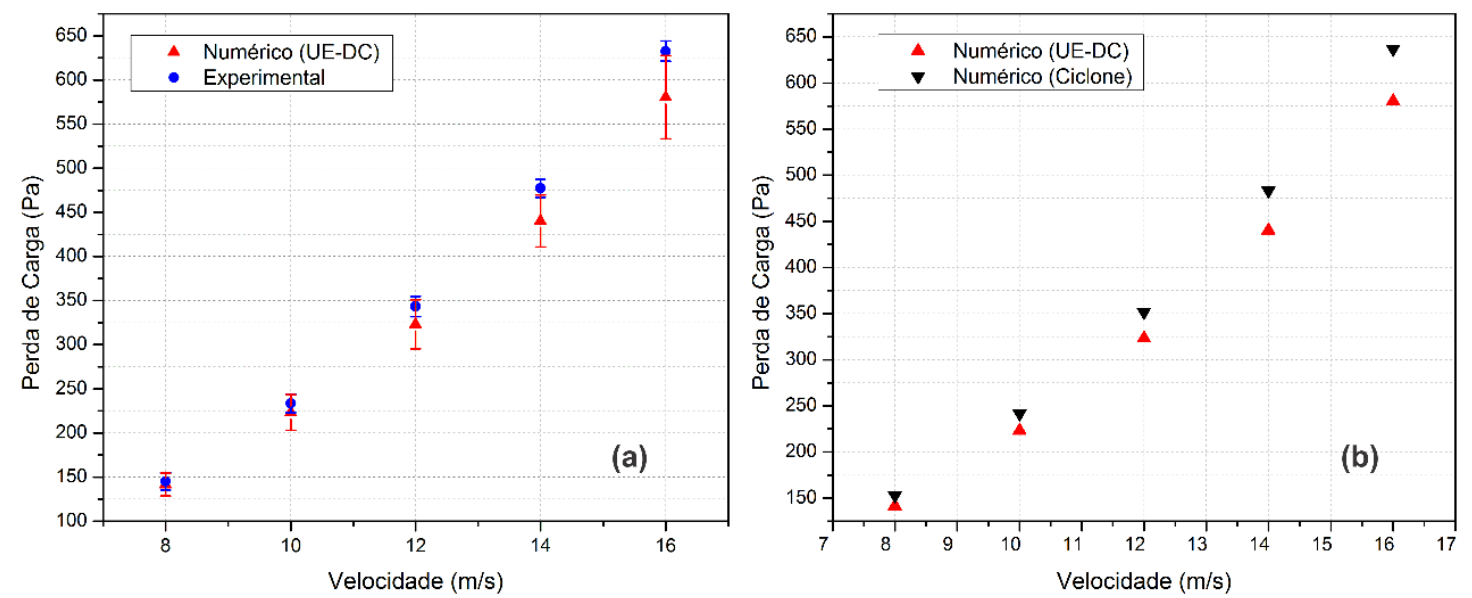

Figura 4: Perda de Carga: (a) comparação da perda de caga experimental e numérica (UE-DC); (b) comparação da perda de carga para ambas as geometrias simuladas.

\subsection{Estudos de validação da predição dos perfis de velocidade}

A Figura 5 apresenta os resultados dos perfis de velocidade axial e tangencial obtidos experimentalmente com o sistema Stereo PIV e os obtidos através da simulação numérica para a geometria da UE-DC, utilizando velocidade na entrada do ciclone em $12 \mathrm{~m} / \mathrm{s}$. Esta ainda mostra a barra do desvio padrão dos experimentos realizados com o Stereo PIV e a barra da incerteza numérica obtida com o método GCI. Portanto, a partir da Figura 5 (a) observou-se o perfil de velocidade axial numérico representou bem os dados experimentais, uma vez que o erro associado ao desvio padrão dos experimentos físicos e da incerteza numérica se sobrepuseram. Analisandose o perfil de velocidade tangencial (Figura 5 (b)), os resultados não foram tão satisfatórios. Porém, estes também mostraram boa reprodutibilidade dos dados quando avaliado de um ponto de vista qualitativo, já que ambos apresentam as mesmas tendências. 

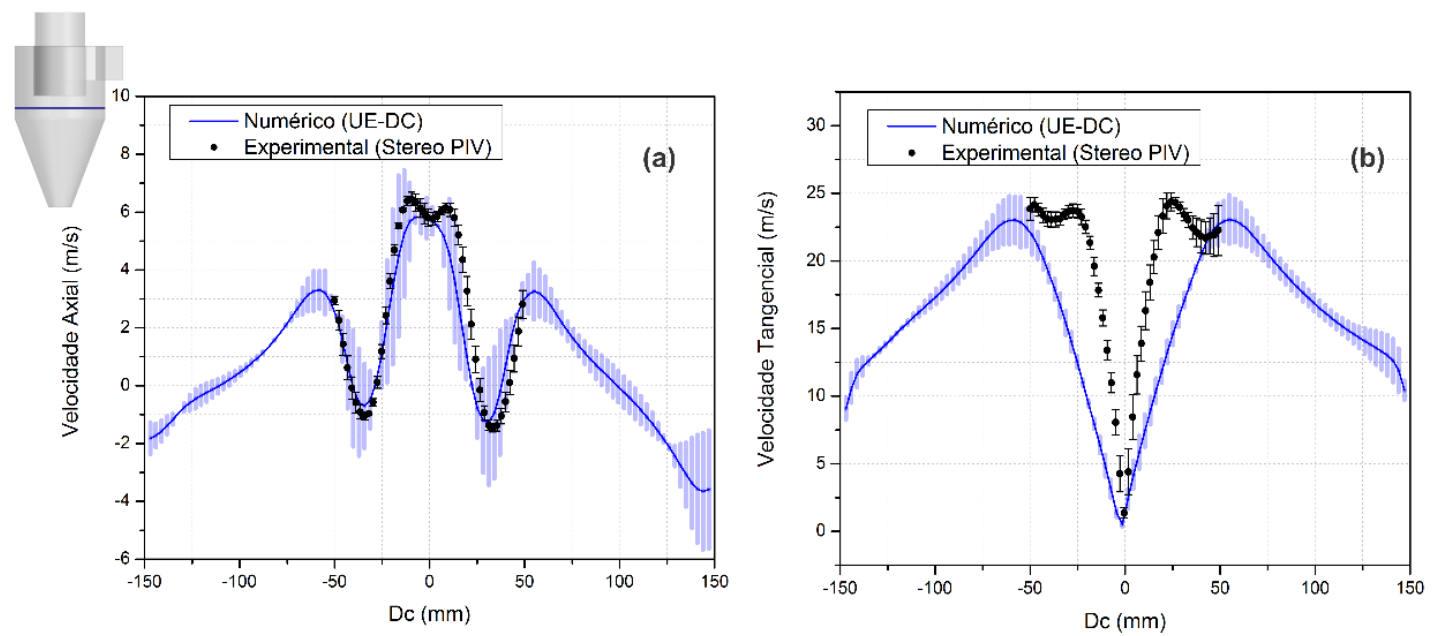

Figura 5: Comparação dos perfis de velocidade experimental e simulado para a geometria da UEDC: (a) perfil de velocidade axial; (b) perfil de velocidade tangencial.

A Figura 6 mostra as diferenças entre os perfis de velocidade axial e tangencial simulados com a geometria da UE-DC e os simulados com a geometria do ciclone, para análise dos efeitos geométricos de entrada e saída no ciclone sobre os perfis de velocidade.
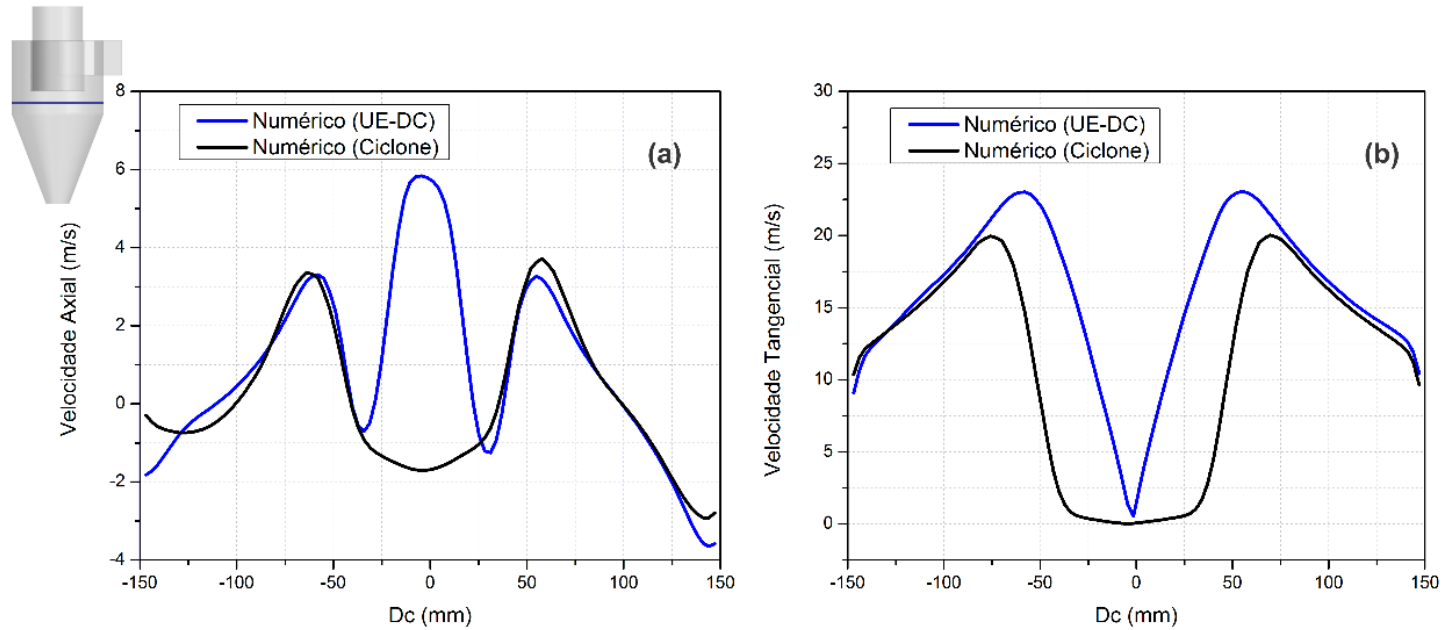

Figura 6: Comparação dos perfis de velocidade simulados para ambas as geometrias: (a) perfil de velocidade axial; (b) perfil de velocidade tangencial.

Analisando o perfil de velocidade axial da UE-DC, observou-se um pico de velocidade ascendente localizado no eixo central do ciclone, diferentemente do perfil bimodal apresentado pelos ciclones convencionais. Já analisando a velocidade tangencial, o perfil da UE-DC encontrase mais deslocado ao centro do ciclone e com maior magnitude dos picos de velocidade tangencial, quando comparado aos simulados com a geometria do ciclone. Tais comportamentos diferenciados nos perfis de velocidade axial e tangencial podem vir a aumentar ou diminuir a eficiência de coleta.

O comportamento diferenciado do perfil de velocidade axial no ciclone obtido a partir das simulações com a geometria da UE-DC e corroborados a partir de experimentos físicos com o 
sistema Stereo PIV (Figura 5 (a)), foi atribuído aos efeitos de saída do ciclone. Tal comportamento se deve ao fato de que após a saída do ciclone, encontra-se uma redução da seção transversal do duto seguido por uma curva, fazendo com que o escoamento ascendente que saí do ciclone, retorne com reversão parcial do escoamento após este atingir o final da redução do duto de saída.

\section{CONCLUSÕES}

Através dos estudos experimentais e numéricos realizados, concluiu-se que o modelo de turbulência RSM-SSG é adequado para a predição da perda de carga, do perfil de velocidade axial e para a magnitude do pico de velocidade tangencial em ciclones.

Por fim, através da comparação das simulações realizadas com ambas as geometrias, observou-se que a perda de carga no ciclone ficou menor quando simulado toda a unidade experimental (UE-DC); que o perfil de velocidade tangencial para a geometria da UE-DC, obteve os picos com maior velocidade; e que o perfil de velocidade axial da simulação da UE-DC apresentou um vórtice ascendente adicional, sendo este comportamento diferenciado dos perfis bimodais geralmente observados pelos ciclones convencionais. Tais diferenças foram atribuídas aos efeitos geométricos de entrada e saída do ciclone e os mesmos podem trazer consequências ainda não avaliadas à eficiência de coleta.

\section{REFERENCIAS}

BERNARDO, S; PERES, A. P.; MORI, M. Computational Study of Cyclone Flow Fluid Dynamics Using a Different Inlet Section Angle. Thermal Engineering. v. 4, n.1, p. 18-23, 2005.

CERNECKY, J; PLANDOROVA, K. The Effect of the Introduction of an Exit Tube on the Separation Efficiency in a Cyclone. Brazilian J. Chem. Eng. v. 30, n. 3, p. 627-641, 2013.

DIAS, D. B. Avaliação de Técnicas de Fluidodinâmica Computacional em Ciclones. 2009. 104f. Dissertação (mestrado) - Universidade Estadual de Campinas, Campinas, São Paulo. 2009.

EL-BATSH, H. M. Improving Cyclone Performance by Proper Selection of the Exit Pipe. Applied Mathematical Modelling. p. 5286-5303, 2012.

HOFFMANN, A. C.; STEIN, L. E. Gas Cyclones and Swirl Tubes: Principles, Design and Operation. 2nd Edition. Springer. Berlin Heidelberg New York. p. 422. 2008.

NORILER, D. ; VEGINI, A. A. ; SOARES, C. ; BARROS, A. A. C. ; MEIER, H. F. ; MORI, M. A New Role for Reduction in Pressure Drop in Cyclones Using Computational Fluid Dynamics Techniques. Brazilian Journal of Chemical Engineering, Brasil, v. 21, n. 1, p. 93-101, 2004.

SGROTT JR. O. L.; COSTA K. K.; NORILER D.; MARTIGNONI W.P.; MEIER H. F. Cyclones Project Optimization by Combination of an Inequality Constrained Problem and Computational Fluid Dynamics Techniques (CFD), Che. Eng. Trans., v. 32, p. 2011-2016, 2013.

SPEZIALE, C. G.; SARKAR, S.; GATSKI, T. B. Modelling the Pressure-Strain Correlation of Turbulence: An Invariant Dynamical Systems Approach. J. Fluid Mech. v. 227, p. 245-272, 1991. 